\title{
Reply to the Letter to the Editor: infection control protocol inside computed tomography suites during coronavirus disease 2019 outbreak
}

\author{
Tsuneo Yamashiro $^{1}$. Daisuke Utsunomiya ${ }^{1}$
}

Received: 14 April 2020 / Accepted: 16 April 2020 / Published online: 6 May 2020

(C) Japan Radiological Society 2020

I would like to express my gratitude to Dr. Joob and Dr. Wiwanitkit for their thoughtful letter regarding our report [1]. As they suggested, the risk faced by radiologists, radiologic technologists, and nurses who practice in the computed tomography (CT) suites is a critical issue. It is challenging for most hospitals to renovate the CT suite for scanning patients infected with the novel coronavirus, severe acute respiratory syndrome coronavirus 2 (SARS-CoV-2). It is considered that the person-to-person transmission is mainly caused by droplet or contact transmission [2], and donning the appropriate personal protective equipment (PPE) and sanitizing with alcohol-based disinfectants may be adequate to prevent in-CT-suite transmission. In contrast, van Doremalen et al. [3] reported the possibility of aerosol and fomite transmission of SARS-CoV-2. Therefore, when respiratory intensive care, e.g., endotracheal intubation, is performed in the CT suite for a coronavirus disease 2019 patient with serious respiratory insufficiency, the CT suite should be nonoperational for $2-3 \mathrm{~h}$. This measure is for air change to occur after the patient has left the CT suite to avoid aerosol and fomite transmission.
The current situation has changed, and another serious problem has risen. The stock of PPE was sufficient at the time of submission of our report [1], but the PPE shortage has increased the risk of infection for the medical staff. The support of the government may be necessary to address this problem that confronts us.

\section{References}

1. Nakajima K, Kato H, Yamashiro T, Izumi T, Takeuchi I, Nakajima H, et al. COVID-19 pneumonia: infection control protocol inside computed tomography suites. Jpn J Radiol. 2020. https:// doi.org/10.1007/s11604-020-00948-y.

2. Wu Y-C, Chen C-S, Chan Y-J. The outbreak of COVID-19: an overview. J Chin Med Assoc. 2020;83:217-20.

3. van Doremalen N, Bushmaker T, Morris D, Holbrook M, Gamble A, Williamson B, et al. Aerosol and surface stability of SARSCoV-2 as compared with SARS-CoV-1. N Engl J Med. 2020. https ://doi.org/10.1056/NEJMc2004973.

Publisher's Note Springer Nature remains neutral with regard to jurisdictional claims in published maps and institutional affiliations.

Tsuneo Yamashiro

clatsune@yokohama-cu.ac.jp

1 Diagnostic Radiology, Yokohama City University,

3-9, Fukuura, Kanazawa-ku, Yokohama-shi,

Kanagawa 236-0004, Japan 